\title{
Design of Intelligent Water Heater Remote Monitoring and Control System Based on IoT Platform
}

\author{
Lian Xiaoqin*, Wu Wenbo, Zhao Honglin, Hao Baozhi and Gong Yonggang \\ College of Computer and Information Engineering, Beijing Technology and \\ Business University, Beijing 100048, China \\ lianxq@263.net
}

\begin{abstract}
The Internet of Things technology has been developing rapidly in recent years, bringing great convenience to daily life. This article elaborates a solution of the remote monitoring system for intelligent water heater based on Ayla IoT platform. An Ayla module is adopted to connect Ayla cloud services with microcontroller of intelligent water heater. An iOS APP is designed by using Model-View-Controller framework and asynchronous socket technology and provides users an effective way to operate and monitor the intelligent water heater. Moreover, this article presents two methods to verify the accuracy and real-time performance of data transmission in this system.
\end{abstract}

Keywords: Internet of Things, Ayla IoT Platform, Intelligent Water Heater, Remote Control

\section{Introduction}

IoT is an abbreviation of Internet of Things [1], which refers to uniquely identifiable objects (things) and their virtual representations in an Internet-like structure [2]. Nowadays, the development of the IoT leads to the improvement of the traditional technology. Meanwhile, traditional household appliances are improved by being combined with the IoT so as to achieve the purpose of energy conservation, environmental protection and intelligent operation. All sorts of appliances are connected to network which generates a large amount of data from IoT. Through the analysis of the data, the performance of the appliances is enhanced and stability and reliability is improved. These appliances will enable users to enjoy a truly smart life.

A traditional water heater uses an operation panel for control. Users have to be present in order to operate it. Due to the lack of convenience, infrared remote control is added to traditional water heater by some manufacturers in order to facilitate the users' operation. However, a bad user experience may be caused when infrared control is blocked by barriers. Thus, IoT is a principal development direction for intelligent water heater system which brings more comfortable and convenient experience for users.

Ayla Networks [3], from its inception, was founded to enable the IoT. Built for enterprise-scale, Ayla IoT Platform was architected from the ground up to deliver a truly end-to-end software foundation that would enable any device manufacturer to develop not only intelligent products, but also elegant and reliable IoT services for their end users. Ayla IoT Platform consists of three primary components: (1) Ayla Embedded Agents, (2) Ayla Cloud Services, and (3) Ayla Application Libraries.

An intelligent water heater remote monitoring and control system based on Ayla IoT platform is designed in this article. An Ayla module is added to traditional water heater in order to connect it to the Ayla cloud services. Ayla module is a production of Ayla

* Corresponding Author 
embedded agents. An iOS APP using Ayla application libraries is designed. Users can use the APP on mobile client to monitor and control intelligent water heater. In this way, It is more convenient than traditional water heater and can save electric energy.

\section{Overall Design Scheme}

The intelligent water heater remote monitor and control system consists of traditional water heater, Ayla module, home gateway, Ayla cloud services and mobile client. Figure 1 shows the schematic of the intelligent water heater system. The traditional water heater is a conventional electrical water heater without remote control function. Ayla module is used to make traditional water heater connected to home gateway. As connected to the home gateway, the water heater can be controlled by mobile client in two different ways. The first way is to use mobile client to control under local area network (LAN). Control commands from mobile client are sent to Ayla module through home gateway. Then, Ayla module transmits these commands to traditional water heater. The second way is to connect the mobile client to Ayla cloud services under wide area network (WAN) so that the Ayla cloud services can transmit control commands to Ayla module.

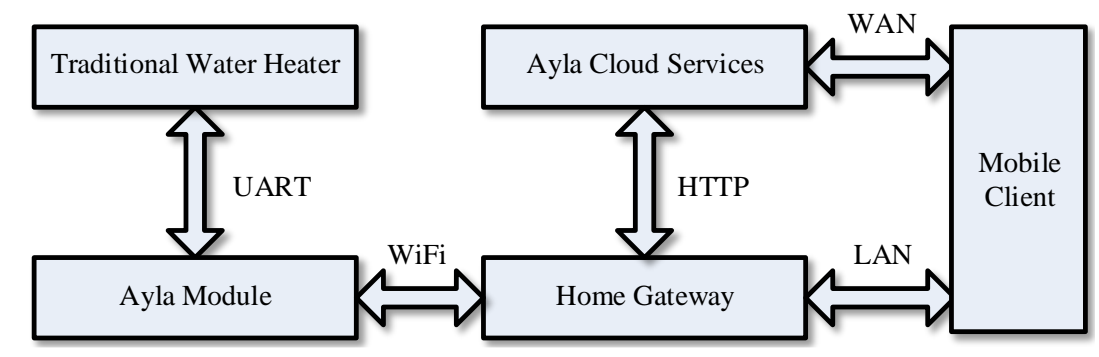

Figure 1. Intelligent Water Heater System Schematic

\section{Hardware Design of System}

This article provides a hardware solution of intelligent water heater which combines traditional water heater and Ayla module. The hardware design of the system is shown in Figure 2. The operation panel is adopted in traditional water heater control pattern. The traditional water heater cannot be controlled remotely. Communication interface has been designed in traditional water heater in order to expand further function. The communication protocol has been provided by manufactures. In this article, Ayla module is added to traditional water heater to make it connected to Internet. Traditional water heater and Ayla module use the communication protocol to exchange information by serial interface. In this way, remote control function of the intelligent water heater will be finally realized.

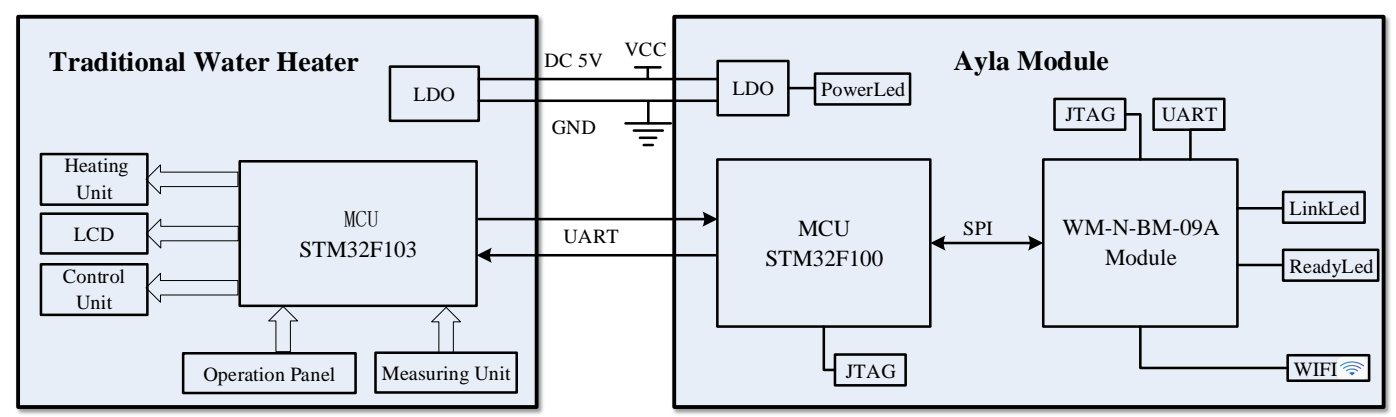




\section{Figure 2. Hardware Design of System}

\subsection{Traditional Water Heater}

As is shown in Figure 2, traditional water heater consists of Low Dropout Regulator (LDO), MCU, heating unit, LCD, control unit, operation panel and measuring unit. Traditional water heater is connected to Ayla module by serial interface. The core of the traditional water heater is microcontroller STM32F103VBT6 which meets the requirement of stability and real-time ability.

By using communication protocol provided by the manufacturer, traditional water heater is able to transfer the following information to Ayla module: machine status, clock information, temperature setting, heating mode and fault notification.

Meanwhile, Ayla module is able to transfer the following control commands to traditional water heater: controlling machine status, clock calibration, setting temperature and controlling heating mode.

\subsection{Ayla Module}

Ayla module which plays a vital role in the whole system makes the traditional water heater connected to home gateway so as to communicate with Ayla cloud services or mobile client.

As is shown in Figure 3, Ayla module mainly consists of LDO, MCU and WM-NBM-09A Module.

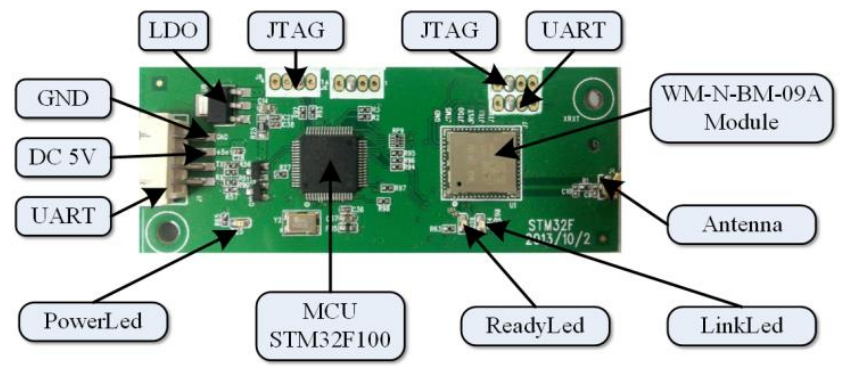

Figure 3. Circuit Board of Ayla Module

Ayla module uses the AMS1117 linear regulator chip which converts voltage from 5 to 3.3. The LDO provides stable working voltage for each chip on the modules. The Power-Led will be bright if LDO works properly.

The MCU is STM32F100RBT6B in Ayla module, which adopts serial communication mode to communicate with traditional water heater. The MCU receives data from traditional water heater by intermittently querying. After data processing, the main control chip transmits valid data to WM-N-BM-09A Module through SPI-bus.

The WM-N-BM-09 wireless SiP module, provided by Ayla Networks, is the connector between the Ayla cloud services and MCU. It consists of a Broadcom BCM43362 single-chip and a STM32F205 microcontroller. As is shown in Figure 4, it has SPI-bus interface. SPI interface which is the full-duplex and three lines synchronous serial peripheral interface was first presented by the Motorola. It adopts the model of master-slave architecture and supports multiple slave devices and usually only has a single master [4]. After being connected to gateway through WiFi, WM-NBM-09A Module processes real-time status data that comes from MCU and sends the processed data to Ayla cloud services or mobile client through home gateway. Thus, users can get real-time status of traditional water heater in mobile client. Meanwhile, 
WM-N-BM-09A Module can get control commands from mobile client or Ayla cloud services and transmit them to MCU by SPI-bus. The control commands in MCU are sent to traditional water heater ultimately. In this way, users can control the traditional water heater in mobile client and the remote control has been realized.

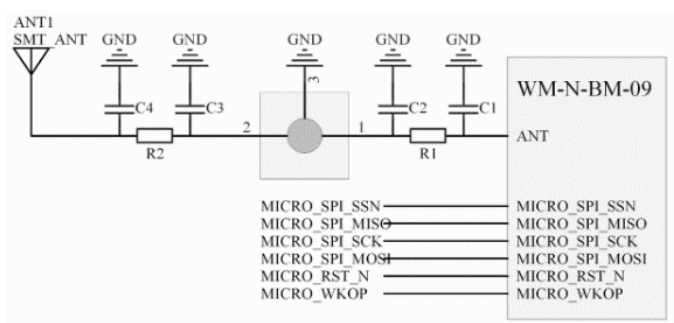

Figure 4. WN-N-BM-09

Moreover, there are two indicator lamps in Ayla module which are Ready-Led and Link-Led, The indicator lamp Ready-Led will be bright if the device operates normally. Only if the device connects to the internet successfully, the indicator lamp Link-Led will be bright.

\section{Software Design of System}

The software design of this system consists of three parts: traditional water heater, Ayla module and APP. The program of traditional water heater is designed by manufacturer. This article focuses on the program design of Ayla module and iOS APP.

\subsection{Software Design of Traditional Water Heater}

The core of the traditional water heater is microcontroller STM32F103VBT6 that has been encapsulated inside the traditional water heater. Its program is designed by manufacturer and it is not the focus of this article. Traditional water heater can be monitored and controlled by Ayla module through serial communication. Ayla module sends control commands to traditional water heater using serial interface. Then, traditional water heater performs control commands.

\subsection{Software Design of Ayla Module}

Program of Ayla module includes MCU program and wireless module program. The program in wireless module which is provided by Ayla Networks can be automatically upgraded through Over the Air Technology (OTA). We concentrate on the design of MCU program in this article. The program of MCU is designed to realize data interaction between SPI interface of wireless module and serial interface of traditional water heater. The control commands from SPI interface is converted to serial communication format according to serial communication protocol. In this way, traditional water heater can be controlled.

The microcontroller of Ayla Module is STM32F100RBT6B. Figure 5 is the flow chart of MCU. After Ayla Module starts working, MCU completes system initialization first and the timer whose timing period is 1 s goes off. Then, MCU waits for interrupt event occurrence. If interrupt event occurs, the MCU runs the corresponding interrupt routine. 


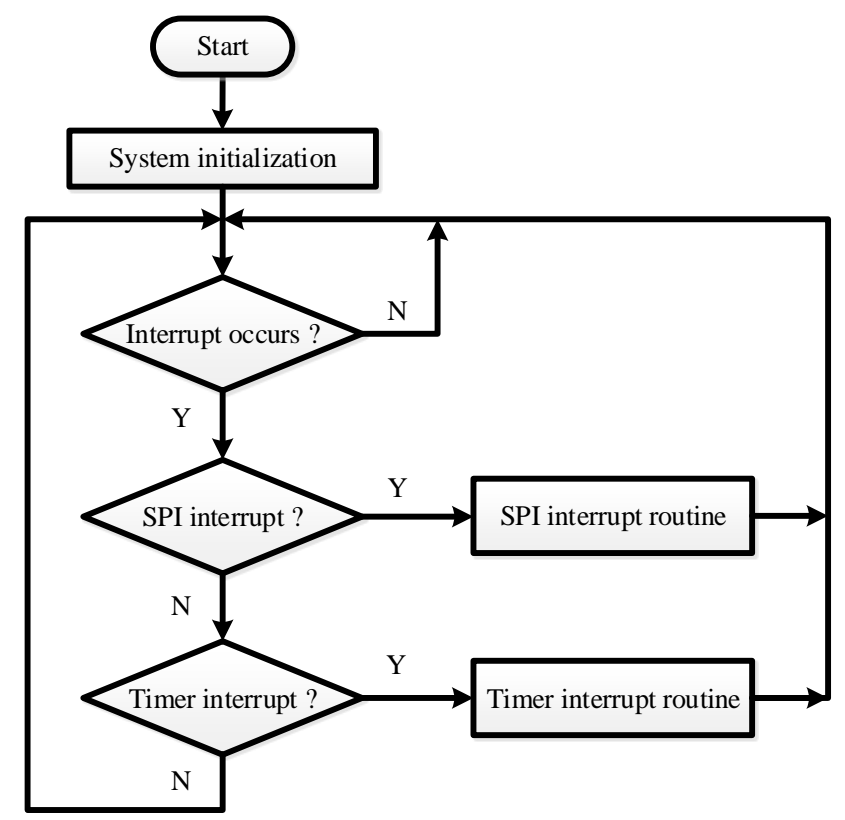

Figure 5. Flow Chart of MCU

Figure 6 is the flow chart of timer interrupt routine. When timer interrupt happens, the microcontroller determines whether to receive the control commands from wireless module or not. If control commands are received, the microcontroller converts and transmits them to traditional water heater. Otherwise, the microcontroller transmits query commands to traditional water heater. 


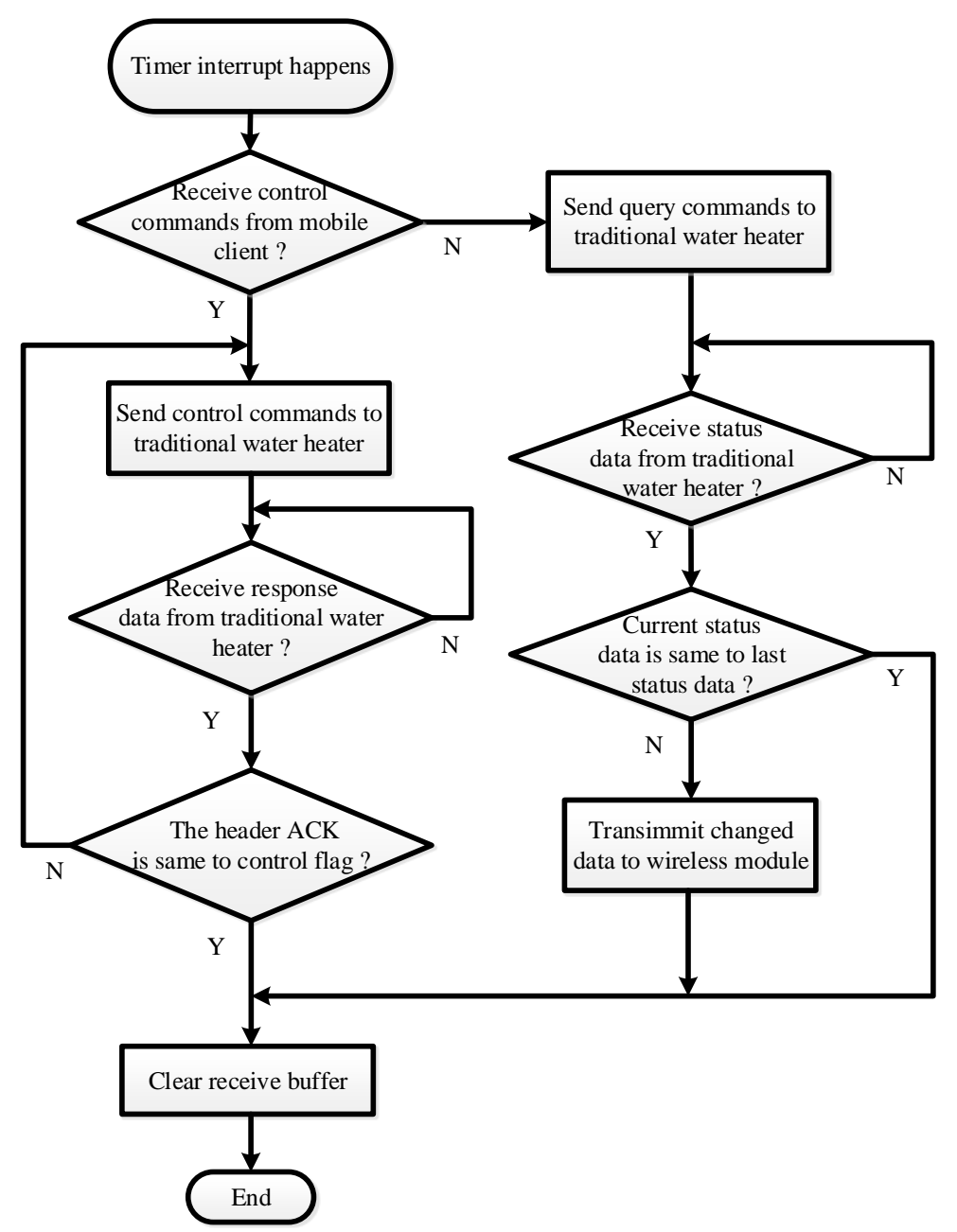

Figure 6. Flow Chart of Timer Interrupt Routine

When Ayla module transmits control commands to traditional water heater, a response data will be sent back from traditional water heater. MCU will verify whether the header acknowledgement character (ACK) of response data is the control flag. If the header ACK is not the same to control flag, Ayla module will re-transmit control commands to traditional water heater.

When Ayla module transmits query commands to traditional water heater, status data from traditional water heater will be sent to Ayla module by serial communication. The status data includes machine status, clock information, temperature setting, heating mode and fault notification. After receiving status data from traditional water heater, the microcontroller starts data verification using CRC check. If a CRC error is detected, the data is discarded. The program function of serial communication is as follows. 


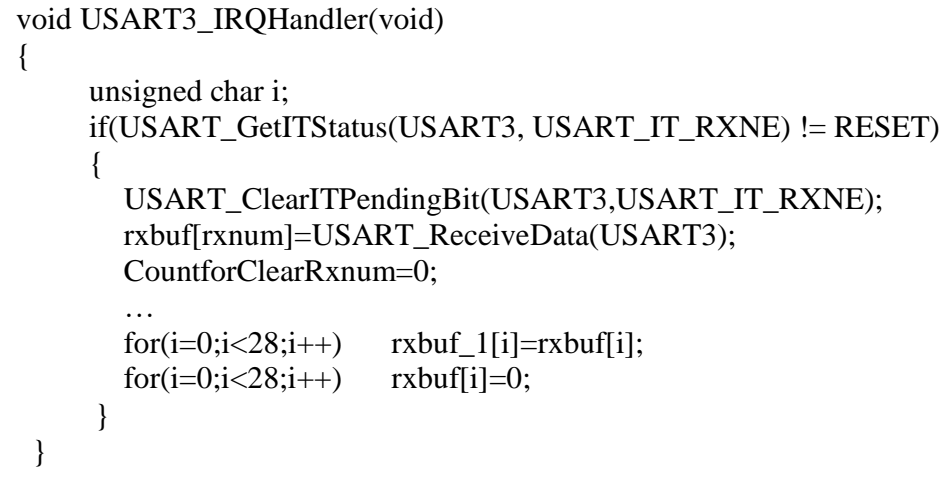

After the CRC check is completed, MCU compares new status data with the status data received last time each time. If the two received status data are not the same, the changed status data will be sent to wireless module through SPI-bus and then sent to mobile client ultimately. Lastly, the receive buffer is cleared for the preparation of receiving new status data. The SPI communication program function of MCU is as follows.

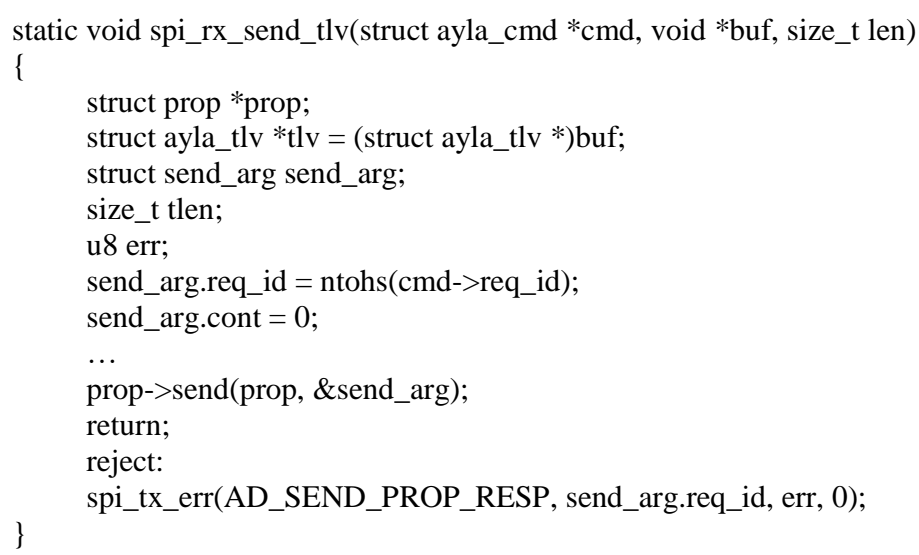

\subsection{Software Design of APP}

The APP of the system is developed on iOS. The iOS is an operating system built into Apple's smart phone iPhone, digital media player, iPod touch and iPad [5]. The iOS is a stable and efficient mobile terminal system. In this article, an APP is designed to control intelligent water heater that consists of traditional water heater and Ayla module. APP uses the communication functions of Ayla application libraries to connect with Ayla cloud services.

4.3.1. Goal of APP Design: APP is designed for users to control intelligent water heater. In the following two cases, the control can be achieved.

The first case is that the intelligent water heater and mobile devices are in the same LAN. The control commands from APP are sent to Ayla module through home gateway. The traditional water heater gets the commands from Ayla module and performs control commands. Meanwhile, the control commands are uploaded to Ayla cloud services and corresponding parameters in Ayla cloud services are modified.

The second case is that the intelligent water heater and mobile devices are in the WAN. The intelligent water heater can be controlled remotely. In this case, APP can send control commands to Ayla cloud services first so that the Ayla cloud services 
sends control commands to intelligent water heater through home gateway. The traditional water heater gets the information from Ayla module and performs operating commands.

4.3.2. Design of APP Mode and Structure: The structure of APP adopts objectoriented structure under the standard Model-View-Controller (MVC) framework which is shown in Figure 7. The model of the framework stores the status parameter of intelligent water heater, the reserved information and the users' information. The view of the framework is the human-APP interaction interface. The controller of the framework is a connector between the model and the view. The status of intelligent water heater extracted from the model is loaded into the view through controller. When the user interacts with the view, the controller is responsible for informing the model of the status updating.

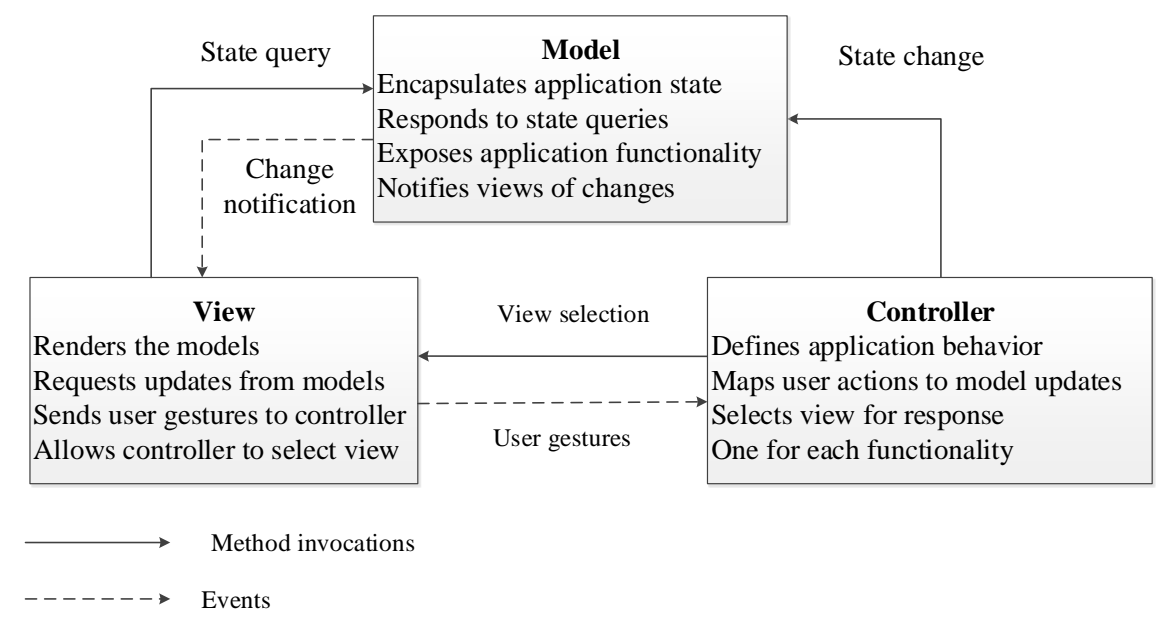

Figure 7. MVC Structure of APP

4.3.3. Communication Mode and Multi-Threaded Design of APP: The core technology in this design is the communication among intelligent water heater, Ayla cloud services and APP. The following section elaborates the communication in two cases of LAN and WAN. Different threads are adopted by APP according to different communication status. After APP's running, it determines what kind of network status is, and then it goes into the corresponding threads and executes code. The thread flow of the APP is shown in Figure 8. 


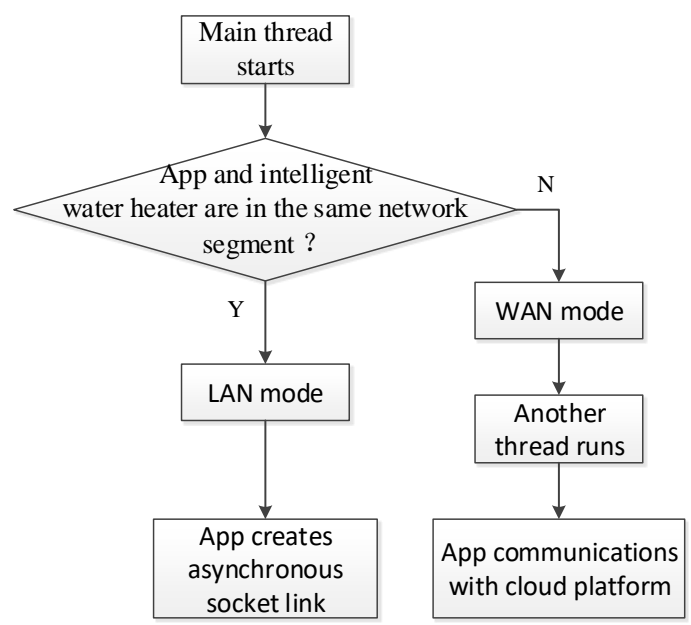

Figure 8. Thread Flow of APP

In the LAN communication mode, APP sends UDP broadcast to search for server. If intelligent water heater and APP are in the same LAN, intelligent water heater will send response information through home gateway [6]. As is shown in Figure 7, the APP creates a socket connection according to UDP protocol. After the connection is established, APP uses asynchronous socket mode and starts the local communication mode.

As traditional socket connection has a low communication rate, process jams will happen. Process will continue to execute until the socket function has returned information. By using asynchronous socket methods, a process called Run-Loop is added to the current thread to monitor the intelligent water heater. At the same time, the main thread continues to perform management UI interface in order to avoid feigning death when APP blocking appears. In this way, the APP can receive the intelligent water heater status in time and can also respond to user interactions at the same time.

If the intelligent water heater status changes, wireless module will send a message to APP. After receiving the message, APP uses GET request mode in HTTP protocol to obtain status variables from intelligent water heater. At the same time, wireless module will also use POST request mode in HTTP protocol to send the messages to Ayla cloud services. APP can also use POST request mode to change the status of the intelligent water heater. If the status changes, corresponding parameters will change in Ayla cloud services.

In the WAN communication mode, APP chooses another thread. Every 5 seconds, it uses GET request mode to contact with Ayla cloud services in order to query the attribute variables that are statuses of intelligent water heater. In the same way, status changes of intelligent water heater will be promptly sent to the Ayla cloud services.

\subsubsection{Preview Effect Diagram of APP:}

While starting this APP, users can log in by inputting the user ID and password. This app can obtain the online status of the intelligent water heater from the Ayla cloud services in the device list interface of the APP. User can observe the real-time status of the intelligent water heater and control it by clicking on the corresponding intelligent water heater on the device list. Figure 9 shows parts of the APP interface screenshot, including the device list interface and the control interface. 


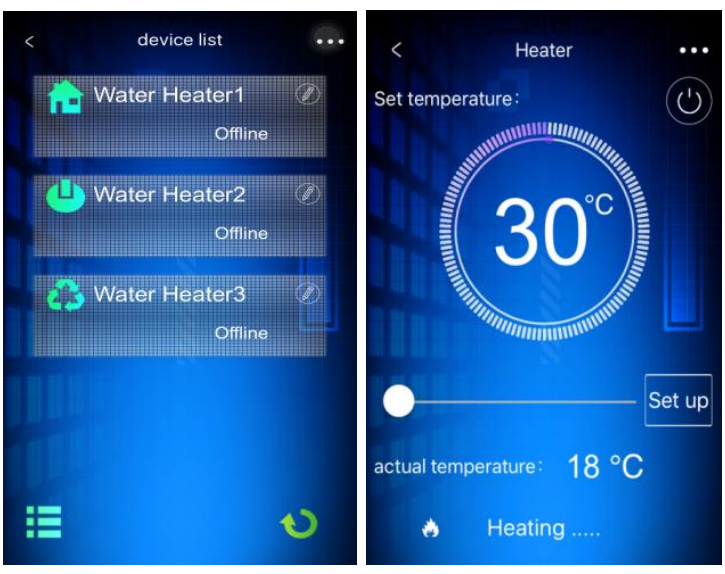

Figure 9. Screenshot of APP Interface

\section{Experimental Results of System}

Architecture diagram of this system is shown in Figure 10. The architecture diagram consists of three parts: water heater, Ayla cloud services and mobile client. In the IoT system, accuracy and real-time requirements of data transmission is an important factor. In order to verify the accuracy and real-time performance of this remote monitoring and control system, we designed the following two methods for performance test in LAN communication mode.

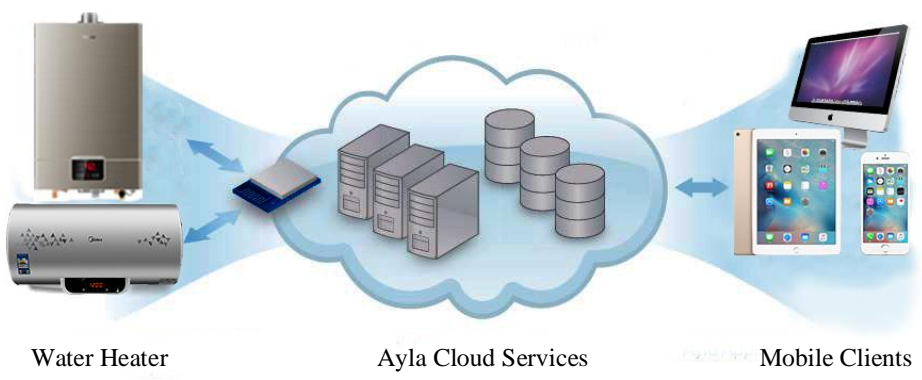

Figure 10. Architecture Diagram of System

The first method uses mobile client to change the temperature setting. The accuracy of data transmission is exemplified by comparison of temperature setting in three parts. And real-time performance of data transmission is exemplified by comparison of the time to receive information in three parts. Figure 11 shows the obtained data that consists of three parts which from top to bottom are the data from mobile client, the data from intelligent water heater and the data form Ayla cloud services. Hexadecimal data which is circled in ellipse is the water temperature setting in intelligent water heater. These hexadecimal data are 40, 43, 46...64, 67 degree centigrade after converted to decimal. In data from Ayla cloud services, the column labeled value is the reverse list of temperature. And column labeled detected is the reverse list of UTC time. The difference between UTC time and Beijing time is 8 hours. 


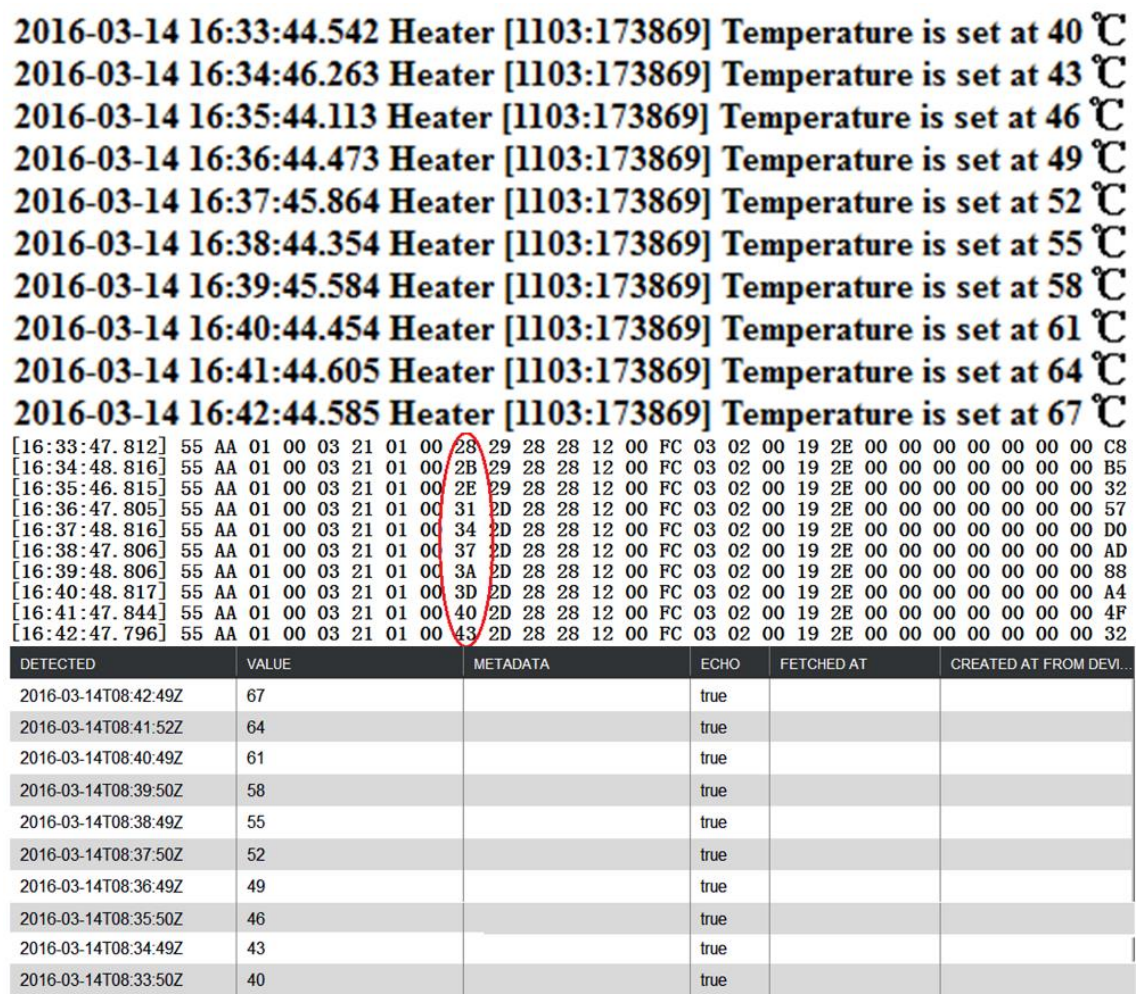

Figure 11. Obtained Data of the First Method

The second method uses operation panel to change the temperature setting. Figure 12 shows the obtained data that consists of three parts which from top to bottom are data from intelligent water heater, data form mobile client and data from Ayla cloud services. Hexadecimal data that are circled in ellipse is the water temperature setting in intelligent water heater. After these hexadecimal data are converted to decimal, the data are $67,64,61 \ldots 43,40$ degree centigrade. 


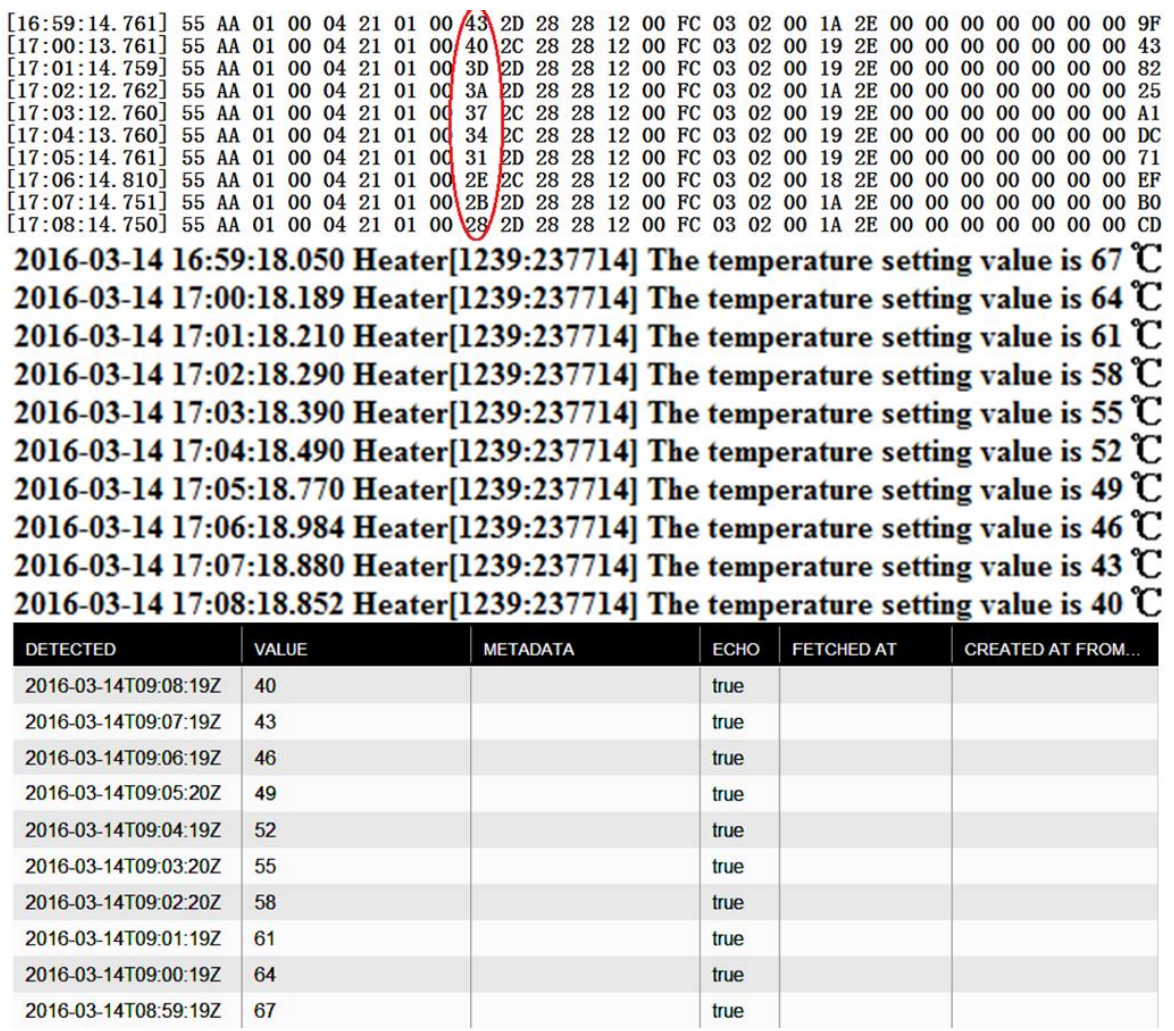

\section{Figure 12. Obtained Data of the Second Method}

From the data it can be concluded that temperature can be transmitted accurately and real-timely. Other parameters can also get accurate and timely transmission. In WAN communication mode, the proposed methods are equally effective.

\section{Conclusion}

The intelligent water heater remote monitoring and control system designed in this article is combined with traditional water heater and Ayla IoT platform. Users can use mobile client to realize remote monitoring anywhere anytime. It not only increases the user experience, but also ensures the intelligent water heater in the best energy saving state.

Using the solutions provided in this article, almost all traditional household appliances can be connected to the IoT. The traditional household appliances do not need many changes as long as the communication interface is reserved and communication protocol is provided. It will make people's life more convenient and help the development of smart home.

\section{Acknowledgements}

This paper was supported by Ayla Networks and the Beijing municipal science and technology of institutions of higher learning and graduate student education innovation and construction project.

\section{Reference}

[1] L. Atzori, A. Iera and G. Morabito, "The Internet of Things: A survey", Computer Networks, vol. 54, (2010), pp. 2787-2805.

[2] Y. Jie, "Smart Home System Based on IoT Technologies", Computational and Information Sciences (ICCIS), 2013 Fifth International Conference on. IEEE, (2013). 
[3] https://www.aylanetworks.com/

[4] S. Sun, J. Ni and Z. Chen, "The design of a DeviceNet-SPI converter module based on the STM32 MCU", Mechatronics and Automation (ICMA), 2015 IEEE International Conference on. IEEE, (2015).

[5] Y. Son, JH. Kim and YS. Lee, "Design and Implementation of the Smart Virtual Machine on iOS Platform for the Mobile Game Portability", International Journal of Smart Home 8.23, (2014).

[6] H.-L. Shang, R.-M. Xu and J.-K. Yuan, "A Smart Home System Based on ZigBee and iOS Software", Parallel and Distributed Systems (ICPADS), 2012 IEEE 18th International Conference on, Singapore, (2012), pp. 940-944.

\section{Authors}

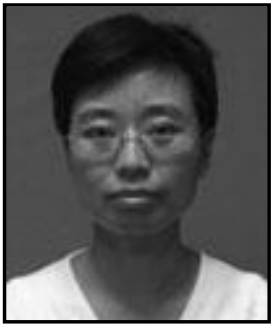

Lian Xiaoqin, she received B.S. degree in Automation from North China University of Technology, M.S. degree in Automation from Beijing Institute of Technology and $\mathrm{Ph}$. D. degree in Mechanical Manufacturing and Automation from Beijing Institute of Technology in 1989, 1992 and 1995 respectively. Her current research interests on electronic commerce website development and mobile development.

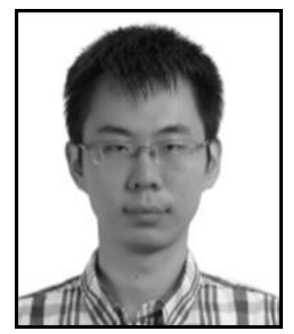

Wu Wenbo, he received B.S. degree in Automation from Beijing Technology and Business University in 2015. He is currently working towards a M.S. degree at Beijing Technology and Business University of Control Engineering. His current research interests on embeded system.

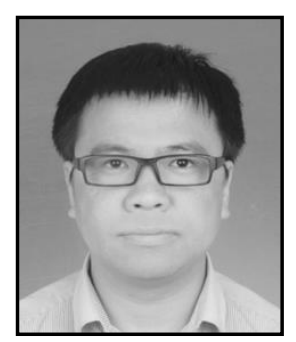

Zhao Honglin, he received B.S. degree in Electronic Information Science and Technology from Shanxi

Datong University in 2013. He is currently working towards a M.S. degree at Beijing Technology and Business University of Detection Technique and Automatic Device. His current research interests on iOS APP design.

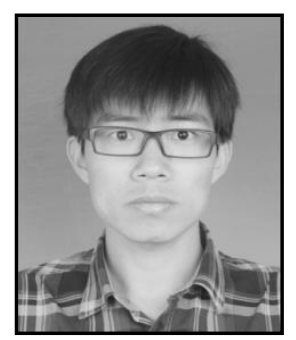

Hao Baozhi, he received B.S. degree in Optoelectronic Technology Science from Hebei Normal University in 2015. He is currently working towards a M.S. degree at Beijing Technology and Business University of Detection Technique and Automatic Device. His current research interests on measurement and control network technology. 


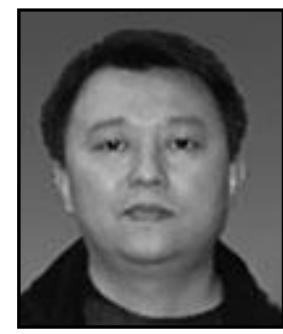

Gong Yonggang, he received B.S. degree in Automation from Guilin University of Electronic Technology and $\mathrm{Ph}$. D. degree in computer application technology from Beijing Institute of Technology in 1995 and 2005 respectively. His current research interests on computer application technology. 